\title{
Optical realization of relativistic non-Hermitian quantum mechanics
}

\author{
Stefano Longhi \\ Dipartimento di Fisica, Politecnico di Milano, Piazza L. da Vinci 32, I-20133 Milano, Italy
}

\begin{abstract}
Light propagation in distributed feedback optical structures with gain/loss regions is shown to provide an accessible laboratory tool to visualize in optics the spectral properties of the one-dimensional Dirac equation with non-Hermitian interactions. Spectral singularities and $\mathcal{P} \mathcal{T}$ symmetry breaking of the Dirac Hamiltonian are shown to correspond to simple observable physical quantities and related to well-known physical phenomena like resonance narrowing and laser oscillation.
\end{abstract}

PACS numbers: 11.30.Er,42.50.Xa

Introduction. Quantum mechanics prescribes that the Hamiltonian $H$ of a physical system must be self-adjoint. Since the the seminal paper by Bender and Boettcher [1], it was realized that the Hermiticity of $H$ can be relaxed, and that a a consistent quantum theory can be constructed for a broader class of Hamiltonians [25]. Among these are parity-time $(\mathcal{P} \mathcal{T})$ Hamiltonians, which possess a real-valued spectrum below a symmetrybreaking point. Non-Hermitian Hamiltonians are also encountered in reduced descriptions of open quantum systems, with important applications to atomic, molecular and condensed-matter physics [6]. In such systems, the lack of Hermiticity can lead to the appearance of exceptional points and spectral singularities, whose physical relevance has been discussed by several authors (see, e.g., [7 [9]). Recently, non-Hermitian extensions of relativistic wave equations [10-12] and non-Hermitian quantum field theories [13] have attracted an increasing interest as well. As some issues in this field are still debated (see, e.g., [13]), physical realizations of non-Hermitian relativistic models remain mostly unexplored. Recently, optical structures in media with a complex refractive index have been proposed to test and visualize non-Hermitian features rooted in the non-relativistic Schrödinger equation with a complex potential [8, 9, 14, 15]. The main motivation in the study of such quantum-optical analogs is that concepts like exceptional points, spectral singularities and $\mathcal{P} \mathcal{T}$ symmetry breaking become measurable quantities in an optical experiment [8, 9]. This has lead to the first experimental visualization of exceptional points and $\mathcal{P} \mathcal{T}$ symmetry breaking in an optical structure [16, 17]. Such results motivate the search for optical simulators of non-Hermitian relativistic wave equations, which is the aim of this Letter. Here it is shown that light propagation in distributed-feedback (DFB) optical structures with gain and/or loss regions, which is at the heart of such important devices as DFB semiconductor lasers [18, 19], can provide a fertile ground to test the spectral properties of non-Hermitian Dirac Hamiltonians. Similarities between light propagation in DFB structures and relativistic wave equations were noticed in early studies on gap solitons in connection with the massive Thirring model of field theory [20], however these previous studies did not consider non-Hermitian interactions.

Non-Hermitian Dirac equation and its optical realiza- tion. Let us consider the Dirac equation in one spatial dimension for a two-component spinor wave function $\psi(x, t)=\left(\psi_{1}, \psi_{2}\right)^{T}$ with time-independent vector $(V)$ and scalar $(S)$ couplings, which in natural units $(\hbar=c=1)$ reads [11, 12]

$$
i \partial_{t} \psi=-i \alpha \partial_{x} \psi+\beta m(x) \psi+V(x) \psi \equiv H \psi
$$

where $\alpha$ and $\beta$ are $2 \times 2$ Hermitian square matrices satisfying the relations $\alpha^{2}=\beta^{2}=1$ and $\alpha \beta+\beta \alpha=0$, $V(x)$ is the time-component of a Lorentzian 2-vector potential, $m(x)$ is the space-dependent effective mass defined by $m(x)=m_{0}+S(x)$, and $m_{0}$ is the rest mass of the Dirac particle. Among the various representations of the Dirac equation, the optical realization of Eq.(1) discussed below is at best highlighted by taking $\alpha=\sigma_{z}$ and $\beta=\sigma_{x}$, where $\sigma_{x}=\left(\begin{array}{ll}0 & 1 \\ 1 & 0\end{array}\right)$ and $\sigma_{z}=\left(\begin{array}{cc}1 & 0 \\ 0 & -1\end{array}\right)$ are the Pauli matrices. If either $V$ or $m$ assume complex values, the Dirac Hamiltonian $H$ is non-Hermitian and its spectrum is, in general, complexvalued. For the Dirac equation, parity and time-reversal operators can be defined as [12] $\mathcal{P}=\mathcal{P}_{0} \beta$ and $\mathcal{T}=\mathcal{K} \beta$, where $\mathcal{P}_{0}$ changes $x$ with $-x$ and $\mathcal{K}$ performs complexconjugation. Hence $\mathcal{P} \mathcal{T} \psi(x)=\psi^{*}(-x)$. $\mathcal{P} \mathcal{T}$ invariance of $H$, i.e. $[\mathcal{P} \mathcal{T}, H]=0$, requires $V(-x)=V^{*}(x)$ and $m(-x)=m^{*}(x)$ [12]. If every eigenfunction of a $\mathcal{P} \mathcal{T}$ invariant Hamiltonian is also an eigenfunction of the $\mathcal{P} \mathcal{T}$ operator, the $\mathcal{P} \mathcal{T}$ symmetry of $H$ is said to be unbroken and its spectrum is real-valued [4]. In the following, we will consider a real-valued effective mass $m(x)$, whereas non-Hermiticity is introduced by allowing the vector potential $V(x)$ to be complex-valued. An optical realization of the Dirac equation (1) is provided by propagation of light waves in an effective one-dimensional DFB structure [18]. Let $n(z)=n_{0}-\Delta n h(z) \cos (2 \pi z / \Lambda+2 \theta(z))$ be the effective index grating of the dielectric structure, where $n_{0}$ is the modal refractive index in absence of the grating, $\Delta n \ll n_{0}$ and $\Lambda$ are the peak index change and the nominal period of the grating, respectively, and $h(z), 2 \theta(z)$ are the normalized amplitude and phase profiles, respectively, of the index grating. The periodic modulation of the refractive index leads to Bragg scattering between two counterpropagating waves at frequencies close to the Bragg frequency $\omega_{B}=\pi c /\left(\Lambda n_{0}\right)$, where $c$ is the speed of 
light in vacuum. The linear space-dependent absorption coefficient of counterpropagating waves in the structure is indicated by $\alpha_{0}(x)\left(\alpha_{0}>0\right.$ in lossy regions, $\alpha_{0}<0$ in gain regions). In a semiconductor DFB structure, gain and loss regions could be tailored by a judicious control of current injection across the active layer [19]. Indicating by $E(z, \tau)=\psi_{1}(z, \tau) \exp \left[-i \omega_{B} \tau+i k_{B} z+i \theta(z)\right]+$ $\psi_{2}(z, \tau) \exp \left[-i \omega_{B} \tau-i k_{B} z-i \theta(z)\right]+$ c.c. the electric field propagating in the DFB structure, where $k_{B}=\pi / \Lambda$, the envelopes $\psi_{1}$ and $\psi_{2}$ of counterpropagating waves satisfy coupled-mode equations [19]. After introduction of the scaled space and time variables $x=z / Z$ and $t=\tau / T$, with $Z=2 n_{0} \Lambda /(\pi \Delta n)$ and $T=Z / v_{g}$, where $v_{g} \simeq c / n_{0}$ is the group velocity of light at frequency $\omega_{B}$, the envelopes $\psi_{1}$ and $\psi_{2}$ satisfy Eq.(1) with a real mass $m$ and a complex-valued vector potential $V$ defined by

$$
m(x)=h(x), \quad V(x)=\frac{d \theta}{d x}-i \gamma(x),
$$

where $\gamma(x)=Z \alpha_{0}(x)$ is the dimensionless absorption coefficient. In the following, we will assume that both $m(x)$ and $V(x)$ have a limited support, i.e. $m=V=0$ for $|x|>L / 2$. This case typically applies to optical DFB structures, in which the grating region is spatially confined to a finite region of length $L$.

Spectral singularities, bound states and resonances of the Dirac Hamiltonian. The spectral properties of the nonHermitian Dirac Hamiltonian $H$, defined by Eqs.(1) and (2), can be investigated by standard methods of scattering theory. To this aim, let us introduce the functions $\phi_{E}^{(1)}(x), \phi_{E}^{(2)}(x), \varphi_{E}^{(1)}(x)$ and $\varphi_{E}^{(2)}(x)$, which satisfy the equation $H \psi=E \psi$ ( $E$ is a complex-valued parameter) with the asymptotic behavior $\phi_{E}^{(1)}=(1,0)^{T} \exp (i E x)$, $\phi_{E}^{(2)}=(0,1)^{T} \exp (-i E x)$ for $x<-L / 2$, and $\varphi_{E}^{(1)}=$ $(1,0)^{T} \exp (i E x), \varphi_{E}^{(2)}=(0,1)^{T} \exp (-i E x)$ for $x>L / 2$. As the Wronskians $W\left\{\phi_{E}^{(1)}, \phi_{E}^{(2)}\right\}=W\left\{\varphi_{E}^{(1)}, \varphi_{E}^{(2)}\right\}=$ 1 do not vanish, $\left\{\phi_{E}^{(1)}, \phi_{E}^{(2)}\right\}$ and $\left\{\varphi_{E}^{(1)}, \varphi_{E}^{(2)}\right\}$ are two sets of linearly-independent solutions to the equation $(E-H) \psi=0$, and therefore there exists a $2 \times 2$ matrix $\mathcal{M}(E)$, with $\operatorname{det} \mathcal{M}(E)=1$, such that

$$
\begin{aligned}
& \phi_{E}^{(1)}(x)=\mathcal{M}_{11}(E) \varphi_{E}^{(1)}(x)+\mathcal{M}_{21}(E) \varphi_{E}^{(2)}(x) \\
& \phi_{E}^{(2)}(x)=\mathcal{M}_{12}(E) \varphi_{E}^{(1)}(x)+\mathcal{M}_{22}(E) \varphi_{E}^{(2)}(x) .
\end{aligned}
$$

Physically, $\mathcal{M}(E)$ is the transfer matrix that connects the amplitudes of forward- and backward-propagating waves from $x=-L / 2$ to $x=L / 2$, which is commonly adopted in the optical context (see, for instance, [19]). The spectral transmission and reflection coefficients, for left $(l)$ and right $(r)$ incidence, can be expressed in terms of the transfer matrix elements in the usual way [9]

$$
t^{(l)}=t^{(r)} \equiv t=\frac{1}{\mathcal{M}_{22}}, \quad r^{(l)}=-\frac{\mathcal{M}_{21}}{\mathcal{M}_{22}}, r^{(r)}=\frac{\mathcal{M}_{12}}{\mathcal{M}_{22}} .
$$

For a $\mathcal{P} \mathcal{T}$-invariant Hamiltonian, one has $\varphi_{E^{*}}^{(1)}(x)=$ $\phi_{E}^{(1) *}(-x)$ and $\varphi_{E^{*}}^{(2)}(x)=\phi_{E}^{(2) *}(-x)$, and thus $\mathcal{M}^{-1}(E)=$
$\mathcal{M}^{*}\left(E^{*}\right)$, which implies $\mathcal{M}_{11}(E)=\mathcal{M}_{22}^{*}\left(E^{*}\right)$.

The spectrum of $H$, as well as the existence of spectral singularities arising from the non-Hermiticity of $H$ [9, 21], can be determined by an inspection of the singularities and branch cuts of the resolvent $G(E)=(E-$ $H)^{-1}$, which takes the integral form [21] $G(E) \psi(x)=$ $\int d y \mathcal{G}(x, y ; E) \psi(y)$, where $\mathcal{G}(x, y ; E)$ is the Green function. Its explicit form reads $\mathcal{G}(x, y ; E)=\mathcal{G}_{+}(x, y ; E)$ for $\operatorname{Im}(E)>0$ and $\mathcal{G}(x, y ; E)=\mathcal{G}_{-}(x, y ; E)$ for $\operatorname{Im}(E)<0$, where

$$
\begin{array}{r}
\mathcal{G}_{+}(x, y ; E)=-\frac{i}{\mathcal{M}_{22}(E)}\left[\Phi(y-x) \phi_{E}^{(2)}(x) \varphi_{E}^{(1) T}(y)+\right. \\
\left.\Phi(x-y) \varphi_{E}^{(1)}(x) \phi_{E}^{(2) T}(y)\right] \sigma_{x},(5)
\end{array}
$$

$$
\begin{array}{r}
\mathcal{G}_{-}(x, y ; E)=\frac{i}{\mathcal{M}_{11}(E)}\left[\Phi(y-x) \phi_{E}^{(1)}(x) \varphi_{E}^{(2) T}(y)+\right. \\
\left.\Phi(x-y) \varphi_{E}^{(2)}(x) \phi_{E}^{(1) T}(y)\right] \sigma_{x},
\end{array}
$$

and $\Phi(x)$ is the Heaviside function $[\Phi(x)=0$ for $x<0$, $\Phi(x)=1$ for $x>0$ ]. On the basis of Eqs.(5) and (6), the following results hold for the spectral properties of $H$.

(i) Continuous spectrum. The continuous spectrum of $H$ is the entire real energy axis $(-\infty<E<\infty)$, where $G(E)$ has a branch cut.

(ii) Point spectrum. The zeros of $\mathcal{M}_{22}(E)$ on the $\operatorname{Im}(E)>0$ half plane, together with the zeros of $\mathcal{M}_{11}(E)$ on the $\operatorname{Im}(E)<0$ half plane, define the point spectrum of $H$; at such energies, the function $\phi_{E}^{(2)}(x)[$ for $\operatorname{Im}(E)>0]$ and $\phi_{E}^{(1)}(x)[$ for $\operatorname{Im}(E)<0]$ are bound states of $H$.

(iii) Resonances. The zeros of $\mathcal{M}_{22}(E)\left[\mathcal{M}_{11}(E)\right]$ on the $\operatorname{Im}(E)<0[\operatorname{Im}(E)>0]$ half plane, i.e. the poles of the analytic continuation of $\mathcal{G}_{+}\left[\mathcal{G}_{-}\right]$on the $\operatorname{Im}(E)<0$ $[\operatorname{Im}(E)>0]$ half plane, correspond to the resonances [antiresonances] of the scattering problem.

(iv) Spectral singularities. A spectral singularity [21] is any zero $E=E_{0}$ on the real axis of either $\mathcal{M}_{11}(E)$ or $\mathcal{M}_{22}(E)$, around which $G(E)$ is unbounded (for either $E=E_{0}+i 0^{+}$or $\left.E=E_{0}-i 0^{+}\right)$in spite of the fact that $E=E_{0}$ does not belong to the point spectrum of $H$.

Note that, for a $\mathcal{P} \mathcal{T}$-invariant Hamiltonian, $\mathcal{M}_{11}(E)=$ $\mathcal{M}_{22}^{*}\left(E^{*}\right)$ and hence at a spectral singularity both $\mathcal{M}_{11}$ and $\mathcal{M}_{22}$ vanish, the resolvent is unbounded for $E=$ $E_{0} \pm i 0^{+}$, and the transmission and reflection spectral coefficients diverge according to Eq.(4) [22]. For such a reason, in Ref. 9] spectral singularities were identified with zero-width resonances. However, for a $\mathcal{P} \mathcal{T}$-non-invariant Hamiltonian, a spectral singularity could arise from the vanishing of $\mathcal{M}_{11}$ but not of $\mathcal{M}_{22}$, i.e. it might not correspond to a divergence of the spectral transmission or reflection coefficients. Such a case was not noticed in Ref. 9] and has a different physical meaning: at such a spectral singularity the potential becomes reflectionless for simultaneous excitation with two waves of same energy $E_{0}$ and amplitudes 1 (for the wave incident from the left side) and $\mathcal{M}_{21}$ (for the wave incident from the right 

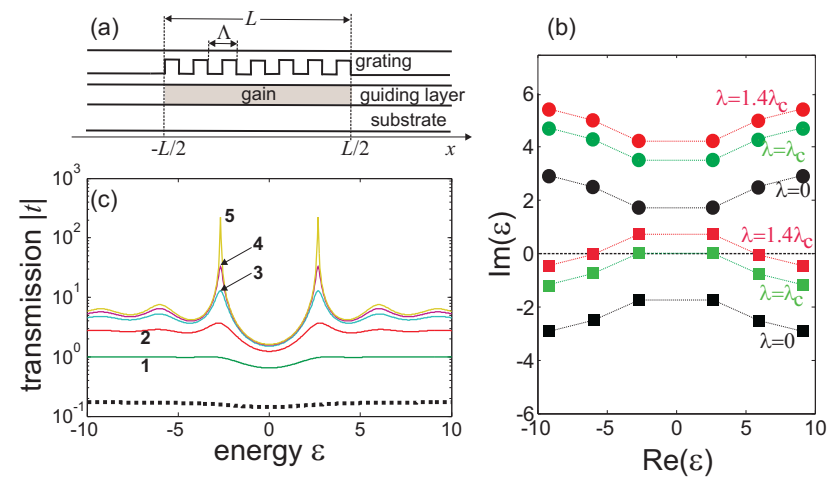

FIG. 1: (color online) (a) Schematic of an optical DFB structure consisting of a uniform index grating with a homogeneous gain region that realizes a $\mathcal{P} \mathcal{T}$-non-invariant Dirac Hamiltonian. (b) Zeros of $\mathcal{M}_{22}$ (squares) and of $\mathcal{M}_{11}$ (circles) in the complex energy plane $\epsilon=E L$ for $m_{0} L=1$ and for increasing values of normalized gain $\lambda$. The critical value $\lambda_{c}$, above which bound states emerge and the spectrum of $H$ ceases to be real-valued, is $\lambda_{c} \simeq 1.755 / L$. (c) Spectral transmission $|t|$ versus normalized energy $\epsilon$ of incident wave for increasing values of $\lambda L$ : Curve $1, \lambda L=0$; curve $2, \lambda L=1$; curve 3 , $\lambda L=1.5$; curve $4, \lambda L=1.65$; curve $5, \lambda L=1.74$. The dotted curve in (c) is the spectral transmission of the DFB structure for $\lambda L=1.74$ when the gain region is replaced by a lossy region.
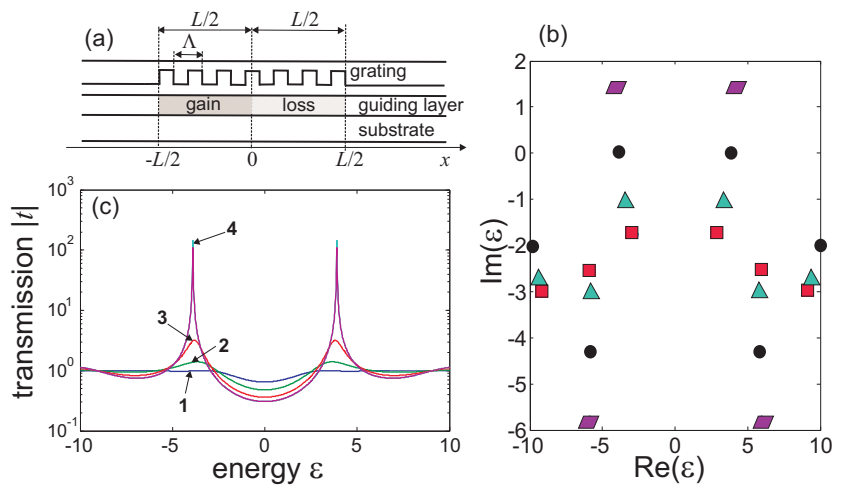

FIG. 2: (color online) (a) Schematic of an optical DFB structure consisting of a uniform index grating with two homogeneous gain and lossy regions that realizes a $\mathcal{P} \mathcal{T}$-invariant Dirac Hamiltonian. (b) Zeros of $\mathcal{M}_{22}$ in the complex energy plane $\epsilon=E L$ for $m_{0} L=1$ and for increasing values of $\lambda$ (squares: $\lambda=0$; triangles: $\lambda L=3$; circles: $\lambda L=4.46$; rhombs: $\lambda L=6)$. $\mathcal{P} \mathcal{T}$ symmetry breaking is reached at $\lambda_{c} \simeq 4.46 / L$. (c) Behavior of spectral transmission $|t|$ for increasing values of $\lambda L$ : Curve $1, \lambda L=0$; curve $2, \lambda L=3$; curve $3, \lambda L=4$; curve $4, \lambda L=4.45$.

side). An example of such a spectral singularity, arising from the crossing of an antiresonance with the real energy axis, is discussed in the first example below.

Optical realizations of spectral singularities and $\mathcal{P} \mathcal{T}$ symmetry breaking. Let us specialize the general results of the spectral theory by considering two examples of complex potentials, in which spectral singularities and
$\mathcal{P} \mathcal{T}$ symmetry breaking of the Dirac equation correspond to well-known physical phenomena in the theory of DFB optical systems.

As a first example, let us consider a $\mathcal{P} \mathcal{T}$-non-invariant potential corresponding to $\theta(x)=0$ and $m(x)=m_{0}$, $\gamma(x)=-\lambda$ in the interval $|x|<L / 2$ [Fig.1(a)], where $\lambda$ is the dimensionless gain coefficient. This case corresponds to the simplest version of a DFB laser with a uniform index grating and a uniform gain region [19]. The transfer matrix of this structure is given by [19]

$\mathcal{M}=\left(\begin{array}{cc}\cosh (\rho L)-i \frac{\sigma}{\rho} \sinh (\rho L) & -i \frac{m_{0}}{\rho} \sinh (\rho L) \\ i \frac{m_{0}}{\rho} \sinh (\rho L) & \cosh (\rho L)+i \frac{\sigma}{\rho} \sinh (\rho L)\end{array}\right)$

where $\sigma \equiv-E+i \lambda$ and $\rho=\left(m_{0}^{2}-\sigma^{2}\right)^{1 / 2}$. Note that the functional dependence of $\mathcal{M}$ on $E$ and $\lambda$ is solely via $\sigma=-E+i \lambda$, so that $\mathcal{M}_{i k}(E, \lambda)=\mathcal{M}_{i k}(E-i \lambda, 0)$. In the Hermitian limit $\lambda=0$, the spectrum of $H$ is purely continuous, and a number of resonances (i.e. zeros of $\mathcal{M}_{22}$ in the $\operatorname{Im}(E)<0$ half plane) as well as of anti-resonances (i.e. zeros of $\mathcal{M}_{11}$ in the $\operatorname{Im}(E)>0$ half plane) do exist [Fig.1(b)]. As $\lambda$ is increased, the spectrum remains real-valued, the resonances (antiresonances) rigidly shift parallel to the imaginary axis, until at a critical value $\lambda=\lambda_{c}$ two resonances cross the real energy axis, i.e. they become two spectral singularities [Fig.1(b)]. The critical value $\lambda_{c}$ can be expressed in the form $\lambda_{c}=f\left(m_{0} L\right) / L$, where the function $f\left(m_{0} L\right)$ can be calculated numerically. For instance, for the case of Fig.1 $\left(m_{0} L=1\right)$ one has $\lambda_{c} L \simeq$ 1.755. At $\lambda>\lambda_{c}$ the resonances cross the real energy axis and bound states with $\operatorname{Im}(E)>0$ emerge; correspondingly, the spectrum of $H$ ceases to be real-valued. Such a transition, from $\lambda<\lambda_{c}$ to $\lambda>\lambda_{c}$, is accompanied by a narrowing of the resonance widths in the transmission spectrum as $\lambda \rightarrow \lambda_{c}^{-}$[see Fig.1(c)] and to the threshold for self-oscillation at $\lambda=\lambda_{c}$. Note that the imaginary parts of the eigenvalues $E$ for $\lambda>\lambda_{c}$ are precisely the growth rates of the two detuned unstable modes at the onset of lasing found in the theory of DFB lasers with a uniform grating [18, 19]. It is remarkable that the well-known physical phenomenon of self-oscillation in DFB lasers mimics the onset of a spectral singularity of a non-Hermitian relativistic wave equation. Let us consider now the same DFB structure of Fig.1(a) but with $\gamma(x)=\lambda$, corresponding to a uniform grating with a homogeneous lossy region. The transfer matrix of the structure is given again by Eq.(7), but with $\sigma=-E-i \lambda$. In this case, as the loss coefficient $\lambda$ is increased from zero, the spectrum remains real-valued shifting parallel to the imaginary axis. At the critical value $\lambda=\lambda_{c}$, two antiresonances (rather than resonances) now cross the real energy axis, i.e. they become two spectral singularities. As opposed to the previous case, at $\lambda=\lambda_{c}$ the spectral transmission does not diverge because the spectral singularities are born from antiresonances (rather than from resonances); see the dotted curve in Fig.1(c). Moreover, the crossing does not correspond, 
as in the previous case, to the onset of lasing in the DFB structure, because at $\lambda>\lambda_{c}$ the two bound states supported by the Hamiltonian have now a negative growth rate $(\operatorname{Im}(E)<0)$, i.e. any initial perturbation (starting from spontaneous emission noise) is damped rather than amplified.

As a second example, let us propose a $\mathcal{P} \mathcal{T}$-invariant DFB structure which could be used to observe $\mathcal{P} \mathcal{T}$ symmetry breaking of the Dirac equation. The structure, schematically shown in Fig.2(a), is composed by a uniform index grating with two symmetric homogeneous gain and lossy regions, i.e. $\theta(x)=0, m(x)=m_{0}$ for $|x|<L / 2, \gamma(x)=-\lambda$ for $-L / 2<x<0$, and $\gamma(x)=\lambda$ for $0<x<L / 2$. The transfer matrix of the structure can be calculated as $\mathcal{M}=\mathcal{M}_{2} \mathcal{M}_{1}$, where $\mathcal{M}_{1}$ and $\mathcal{M}_{2}$ are the transfer matrices of the uniform gain and lossy sections [see Eq.(7)]. Figure 2(b) shows the loci of zeros of $\mathcal{M}_{22}$ in the complex energy plane for increasing values of $\lambda L$ and for $m_{0} L=1$. Note that, owing to the $\mathcal{P} \mathcal{T}$-invariance of $H, \mathcal{M}_{11}(E)=\mathcal{M}_{22}^{*}\left(E^{*}\right)$, and thus the zeros of $\mathcal{M}_{11}$ (not shown in the figure) are simply the complex conjugates of those of $\mathcal{M}_{22}$. As $\lambda$ is increased above the critical value $\lambda_{c} \simeq 4.46 / L$, a $\mathcal{P T}$ symmetry breaking occurs, with the appearance of two pairs of complex-conjugate eigenvalues belonging to the point spectrum of $H$ and arising from two couples of resonances and antiresonances crossing the real energy axis. As the point of $\mathcal{P} \mathcal{T}$ symmetry breaking is approached, narrowing of the transmission resonances is observed [see Fig.2(c)], and the onset of lasing at $\lambda=\lambda_{c}^{+}$ corresponds to the breaking of the $\mathcal{P} \mathcal{T}$ symmetry.

Conclusions. DFB optical structures provide a fertile classical simulator of non-Hermitian relativistic wave equations. This work suggests that well-known phenomena occurring in DFB structures, like spectral narrowing of resonances and self-oscillation, are the measurable quantities associated to the onset of spectral singularities and $\mathcal{P} \mathcal{T}$ symmetry breaking of Dirac Hamiltonians with certain complex couplings.

Work supported by the italian MIUR (Grant No. PRIN-2008-YCAAK, "Analogie ottico-quantistiche in strutture fotoniche a guida d'onda").
[1] C.M. Bender and S. Boettcher, Phys. Rev. Lett. 80, 5243 (1998)

[2] C. M. Bender, D.C. Brody, and H. F. Jones, Phys. Rev. Lett. 89, 270401 (2002).

[3] A. Mostafazadeh, J. Math. Phys. 43, 2814 (2002).

[4] C.M. Bender, Rep. Prog. Phys. 70, 947 (2007).

[5] A. Mostafazadeh, e-print arXiv:0810.5643.

[6] N. Moiseyev, Phys. Rep. 302, 211 (1998); J.G. Muga, J.P. Palao, B. Navarro, and I.L. Egusquiza, Phys. Rep. 395, 357 (1998); I. Rotter, J. Phys. A 42, 1 (2009).

[7] M.V. Berry, Czech. J. Phys. 54, 1039 (2004).

[8] S. Klaiman, U. Günther, and N. Moiseyev, Phys. Rev. Lett. 101, 080402 (2008).

[9] A. Mostafazadeh, Phys. Rev. Lett. 102, 220402 (2009).

[10] C. Mudry, B.D. Simons, and A. Altland, Phys. Rev. Lett. 80, 4257 (1998); H. Egrifes and R. Sever, Phys. Lett. A 344, 117 (2005); A. Sinha and P. Roy, Mod. Phys. Lett. A 20, 2377 (2005); C.S Jia and A. de Souza Dutra, J. Phys. A 39, 11877 (2006); F. Cannata and A. Ventura, Phys. Lett. A 372, 941 (2008); O. Mustafa, S.H. Mazharimousavi, Int. J. Theor. Phys. 47, 1112 (2008).

[11] C.-S. Jia and A. de Souza Dutra, Ann. Phys. 323, 566 (2008); C-S. Jia, P.-Q. Wang, J.-Yi Liu, and S. He, Int. J. Theor. Phys. 47, 2513 (2008).

[12] V.G.C.S. dos Santos, A. de Souza Dutra, and M.B. Hott, Phys. Lett. A 373, 3401 (2009); F. Cannata and A. Ventura, J. Phys. A 43, 075305 (2010).

[13] C.M. Bender, K.A. Milton, and Van M. Savage, Phys. Rev. D 62, 085001 (2000); C.M. Bender, D.C. Brody, and H.F. Jones, Phys. Rev. Lett. 93, 251601 (2004); C.M. Bender, S.F. Brandt, J.-H. Chen, and Q. Wang, Phys. Rev. D 71, 065010 (2005); A. Mostafazadeh, Int. J. Mod.
Phys. A 21, 2553 (2006).

[14] A. Ruschhaupt, F. Delgado, and J. G. Muga, J. Phys. A 38, L171 (2005).

[15] R. El-Ganainy, K. G. Makris, D. N. Christodoulides, and Z. H. Musslimani, Opt. Lett. 32, 2632 (2007); K.G. Makris, R. El-Ganainy, D.N. Christodoulides, and Z.H. Musslimani, Phys. Rev. Lett. 100, 103904 (2008); S. Longhi, Phys. Rev. Lett. 103, 123601 (2009).

[16] A. Guo, G.J. Salamo, D. Duchesne, R. Morandotti, M. Volatier-Ravat, V. Aimez, G. A. Siviloglou, and D. N. Christodoulides, Phys. Rev. Lett. 103, 093902 (2009).

[17] C.E. Rüter, K.G. Makris, R. El-Ganainy, D.N. Christodoulides, M. Segev, and D. Kip, Nature Phys. 6, 192 (2010).

[18] H. Kogelnik and C.V. Shank, J. Appl. Phys. 43, 2327 (1972).

[19] J. Carroll, J. Whiteaway, and D. Plumb, Distributed feedback semiconductor lasers (The Institution of Electrical Engineers, London, 1998).

[20] A.B. Aceves and S. Wabnitz, Phys. Lett. A 141, 37 (1989); D.N. Christodoulides and R.I. Joseph, Phys. Rev. Lett. 62, 1746 (1989).

[21] M. A. Naimark, Linear differential operators (Ungar, New York, 1968); I.-P. P. Syroid, Ukrainian Math. J. 38, 305 (1986); G.S. Guseinov, Pramana 73, 587 (2009).

[22] Unitarity of the scattering matrix, implying $|t|^{2}+$ $\left|r^{(l, r)}\right|^{2}=1$ and thus bounded values of transmission and reflection coefficients, is satisfied for Hermitian Hamiltonians, however in non-Hermitian systems unitarity may be broken [see, for instance: F. Cannata, J.-P. Dedonder, and A. Ventura, Ann. Phys. 322, 397 (2007)]. 